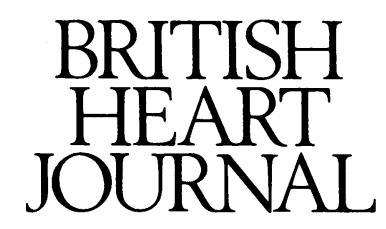

Editorial

\title{
HIV cardiomyopathy: A dark cloud with a silver lining?
}

A wide variety of cardiac problems have been reported in patients infected with the human immunodeficiency virus (HIV). These include pericardial effusion, pulmonary hypertension and right heart failure, infective endocarditis, and tumours such as lymphoma and Kaposi's sarcoma. ${ }^{1}$ However, left ventricular dysfunction caused by myocarditis or cardiomyopathy is emerging as the most important cardiac manifestation of HIV infection. ${ }^{2}$

Retrospective analysis of necropsy studies showed evidence of clinically unsuspected dilated cardiomyopathy in up to $25 \%{ }^{2}$ and of myocarditis in up to $52 \%{ }^{3}$ of patients with the acquired immunodeficiency syndrome (AIDS). Similarly, echocardiography showed impaired myocardial function in up to $75 \%$ of patients with AIDS. ${ }^{4}$ The reported incidence of these phenomena varies widely, probably reflecting differences in methodology as well as the patients' risk group, stage of disease, and local environmental factors; nevertheless, it seems that HIV cardiomyopathy is a common condition in the later stages of the disease and that it affects patients in all major risk groups. ${ }^{5}$

The increasing success in combating opportunistic infections and slowing the progress of HIV disease itself may mean that many patients who otherwise would have died of non-cardiac illnesses will now survive to develop HIV cardiomyopathy. Indeed, end-organ failure and tumours are likely to become the predominant causes of death in patients infected with HIV. This, combined with the predicted rise in the number of infected individuals, particularly heterosexuals, is likely to lead to a substantial increase in the incidence of HIV cardiomyopathy.

In our experience HIV related myocardial dysfunction may easily be overlooked because of the tendency to attribute symptoms such as breathlessness and fatigue to respiratory disease or anaemia. If nothing else, this denies the victim the opportunity of palliative treatment. The diagnosis is readily made by echocardiography which can show dilatation or impaired contractility or both of either or both ventricles. While isolated right ventricular dysfunction can be caused by cardiomyopathy, it can also be caused by pulmonary hypertension secondary to repeated respiratory infections, ${ }^{6}$ thromboembolic disease, or recurrent pulmonary emboli from intravenous debris acquired through drug abuse.

Endomyocardial biopsy in patients with heart failure associated with HIV infection has been performed in several centres and has identified myocarditis caused by potentially treatable infections with organisms such as Toxoplasma gondii. ${ }^{7}$ The value of cardiac tissue obtained by these means is likely to be enhanced by the application of techniques such as the polymerase chain reaction and in situ hybridisation, which can be used to detect the presence of opportunistic organisms and the human immunodeficiency virus itself.
The pathogenesis of HIV cardiomyopathy, particularly its relation to myocarditis, has not been established. However, it seems likely that myocarditis acts as the substrate for the development of HIV cardiomyopathy and that the two conditions form a continuum. Necropsy studies in patients with HIV cardiomyopathy have shown huge variations in the prevalence of concomitant myocarditis. ${ }^{38}$ This may reflect sampling errors, the difficulty of applying diagnostic criteria based on the combination of myocyte necrosis and an inflammatory infiltrate when the ability to mount an inflammatory response is impaired, and differences in the stage of disease.

Myocarditis may be caused by cell damage caused by activation of the immune system; by opportunistic infection with organisms such as Aspergillus fumigatus, ${ }^{9}$ cytomegalovirus, ${ }^{10}$ and Cryptococcus neoformans ${ }^{11}$; or by the human immunodeficiency virus itself. ${ }^{12}$

Activation of the humoral and/or cellular limbs of the immune system may cause damage directly, ${ }^{3}$ or by means of a bystander effect, ${ }^{13}$ where non-infected cells are destroyed by cytotoxic agents released during the course of an immune response directed against an infected minority cell population (for example, macrophages, $T$ lymphocytes). Autoimmune damage may also occur as a result of alterations in the structure or expression of cardiac antigens. ${ }^{14}$ Finally, some of the agents used to treat HIV associated opportunistic infection or retard the progression of the disease itself may be cardiotoxic; for example, pentamidine has been associated with malignant ventricular arrhythmias. ${ }^{15}$ HIV cardiomyopathy probably represents the end stage of various insults operating concurrently and perhaps synergistically. Clearly there is a need to perform prospective studies to establish the true incidence, pathogenesis, and natural history of the condition.

The relation between myocarditis and idiopathic dilated cardiomyopathy is uncertain though the cardiomyopathy may develop from myocarditis. A similar sequence may occur in HIV myocardial disease. The scourge of syphilis in the early part of this century led to greater understanding of the pathophysiology of cardiovascular disease. HIV cardiomyopathy offers a similar opportunity to unravel the pathogenesis of idiopathic dilated cardiomyopathy. Although HIV cardiomyopathy may not have a unified aetiology, it occurs in a well-defined group of patients who can be studied premorbidly, and the associated viral antigens are highly characterised. We have, therefore, an exciting chance to document the natural history of a new disease and correlate this with external risk factors, individual susceptibility, and immunological events in blood and cardiac muscle.

HIV cardiomyopathy is a dark cloud that threatens to become an ever increasing problem, presenting cardiologists with considerable clinical challenges. This will be 
tempered by a silver lining of opportunities to enhance our knowledge of the mechanisms underlying idiopathic dilated cardiomyopathy.

A J J is supported by the British Heart Foundation.

A J JACOB

Department of Cardiology,

NABOON

Royal Infirmary,

Lauriston Place,

Edinburgh $\mathrm{EH} 39 \mathrm{YW}$

1 Acierno LJ. Cardiac complications in acquired immunodeficiency syndrome (AIDS): a review. J Am Coll Cardiol 1989;13:1144-54.

2 Anderson DW. Emerging patterns of heart disease in human immunodeficiency virus infection. Hum Pathol 1990;21:253-9.

3 Anderson DW, Virmani R, Reilly JM, et al. Prevalent myocarditis at necropsy in the acquired immunodeficiency syndrome. J Am Coll Cardiol 1988;11:792-9.

4 Baroldi G, Corallo S, Moroni M, et al. Focal lymphocytic myocarditis in acquired immunodeficiency syndrome (AIDS): a correlative morphologic and clinical study in 26 consecutive fatal cases. J Am Coll Cardiol 1988;12:463-9.
5 Corallo S, Mutinelli MR, Moroni M, et al. Echocardiography detects myocardial damage in AIDS: prospective study in 102 patients. Eur Heart J 1988;9:887-92.

6 Himelman RB, Dohrmann M, Goodman P, et al. Severe pulmonary hypertension and cor pulmonale in the acquired immunodeficiency syndrome. Am J Cardiol 1989;64:1396-9.

7 Grange F, Kinney EL, Monsuez J-J, et al. Successful therapy for toxoplasma gondii myocarditis in acquired immunodeficiency syndrome. Am Heart 1990;120:443-4.

8 Lewis W. AIDS: cardiac findings from 115 autopsies. Prog Cardiovasc Dis 1989;32:207-15.

9 Cox JN, di Dio F, Pizzolato G-P, Lerch R, Pochon N. Aspergillus endocarditis and myocarditis in a patient with the acquired immunodeficiency syndrome (AIDS). Virchows Arch $[A]$ 1990;417:255-9.

10 Stewart JM, Kaul A, Gromisch DS, et al. Symptomatic cardiac dysfunction in children with human immunodeficiency virus infection. Am Heart $J$ in children with

11 Lafont A, Wolff M, Marche C, Clair B, Regnier B. Overwhelming myocarditis due to cryptococcus neoformans in an AIDS patient. Lancet carditis due to

12 Grody WW, Cheng L, Lewis W. Infection of the heart by the human immunodeficiency virus. Am J Cardiol 1990;66:203-6.

13 Ho DD, Pomerantz RJ, Kaplan JC. Pathogenesis of infection with human immunodeficiency virus. $N$ Engl J Med 1987;317:278-86.

14 Cotton P. AIDS giving rise to cardiac problems. JAMA 1990;263:2149.

15 Stein KM, Haronian H, Mensah GA, Acosta A, Jacobs J, Kligfield P. Ventricular tachycardia and torsades de pointes complicating pentamidine therapy of pneumocystis carinii pneumonia in the acquired immunodeficiency syndrome. Am J Cardiol 1990;66:888-9. 